Hautarzt 2020 $\cdot 71: 732-733$

https://doi.org/10.1007/s00105-020-04640-0

Online publiziert: 6 . Juli 2020

(c) Springer Medizin Verlag GmbH, ein Teil von Springer Nature 2020

Das Eponym „DER Fritsch“ hat 2-fache Bedeutung: es steht einmal für das exzellente Hauptwerk des Autors Peter Fritsch (kurz PF): Dermatologie Venerologie. Grundlagen. Klinik. Atlas; Springer (1983), 1998, 2004 und 2018.

Geboren in Wien, absolvierte PF dort Schule (humanistisches Gymnasium), Studium (Promotion 1965) und Fachausbildung (1972 Facharzt, 1976 Habilitation) der Dermatologie, kurz ein Wiener, der allerdings bald und mehrfach den Schritt in die weite Welt wagte wie nach Birmingham (Prof. Cruickshank), Paris (Prof. Prunières) und mit New Haven (Prof. Lerner) und San Francisco (als „visiting professor" bei seinem Lebensfreund Peter Elias) in die Vereinigten Staaten. Solche Studienaufenthalte zur Horizonterweiterung bei anderen „Kulturen“ ermöglichte PF in der Folge dann auch großzügig seinen jungen MitarbeiterInnen, indem er ihre Sabbaticals im In- und Ausland förderte. Dies war dann auch die Basis für die Karriere etlicher seiner Schüler, die letztendlich Vorstände von großen Kliniken (wie z.B. G. Schuler in Erlangen) und Abteilungen in und außerhalb Österreichs wurden. War allerdings das Dermatologische Zentrum in Innsbruck einmal aufgebaut, wurde der Weg ins und vom Ausland zweidirektional, denn KollegInnen aus zahlreichen Ländern und von verschiedenen Kliniken lernten Studienaufenthalte in Tirol sehr zu schätzen.

Wie aber entsteht ein Meisterwerk wie das eingangs erwähnte „Fritsch Buch“? $\mathrm{Da}$ ist zuallererst einmal die profunde und umfassende Kenntnis des Spezialfaches Dermatologie, erworben durch die jahrzehntelange (27 Jahre als Klinikchef in Innsbruck) Zuwendung zu PatientIn-

Helmut Hintner

Salzburg, Österreich

\title{
Laudatio für Univ. Prof. Dr. Peter Fritsch zum 80. Geburtstag
}

nen. PF war in erster Linie ein exzellenter Kliniker, wobei die Aufzählung von Spezialinteressen für Krankheiten wie die TEN (toxisch epidermale Nekrolyse), das SSSS („staphylococcal scalded skin syndrome"), die Vaskulitis, Techniken wie die Auflichtmikroskopie (PF war einer der Erfinder) und für Therapieformen wie mit Retinoiden nur beispielgebend sein kann. Zum anderen führte die stete Frage nach kausalen Zusammenhängen zur Laborarbeit, damals oft in der Freizeit, wobei auch hier das Spektrum breit von der Gewebekultur bis zur essenziellen Dermatohistopathologie reichte.

Dreihundert originale Publikationen (auch gekrönt mit renommierten Preisen wie Eiselsberg, Hoechst, Aesca und Hebra) und 100 Buchartikel bzw. Übersichtsarbeiten sprechen für des Autors Fleiß. Kennt man allerdings die Zufriedenheit, wenn endlich nach zahllosen Stunden im Labor, Nächten des Schreibens und dem Review-Hick-Hack der druckfrische Sonderdruck vor einem liegt, versteht man auch das Vergnügen bei der publikatorischen Tätigkeit und den steten Antrieb für PF. Alles zusammenfassend, goss drittens die exzeptionelle Meisterschaft der deutschen Sprache durch PF das Werk in die perfekte Form.

Ist es jemanden möglich, den Inhalt des „Fritsch Buches“ zu reproduzieren, besteht sie/er nicht nur die Dermatologiefacharztprüfung mit Bravour, nein sie/er gäbe auch einen ausgezeichneten Vorstand jeder Dermatologischen Klinik in der ganzen Welt ab!

Apropos Wortgewalt und Mutterwitz: Stellen Sie sich vor, PF mit seinem tiefgründigen Humor wäre nicht Hautarzt (in Bezug auf diese Berufswahl lesen Sie bitte im „Untergang des Abendlandes in
Anekdoten“ über Tommy nach - s. unten!), sondern Strafverteidiger, Journalist oder Schriftsteller geworden! Auch dann hätte er Karriere gemacht, und es wären besungene Werke entstanden! So gesehen war es gut, dass sein Lebenswunsch, Pfeifenkopfschnitzer zu werden, nicht in Erfüllung ging! Aber nicht nur das Schaffen des Opus, auch dessen Weitergabe, in verständlicher Form, war PF ein großes Anliegen. Er gründete die florierende „Österreichische Akademie für Dermatologische Fortbildung " und die Alpenländischen Dermatologen Nachmittage brachten stets in großer Zahl Nord- und Südtiroler sowie Vorarlberger niedergelassene Hautärzte in Innsbruck zusammen. Hier ist zu erwähnen, dass bei den ,vergnüglichen“ Abschlüssen der Veranstaltungen „der Chef" keineswegs ein Außenseiter, sondern er immer im Brennpunkt war; der Genuss des Lebens war und ist ihm nicht fremd.

Die „Must's“ der akademischen Dermatologie erfüllte PF wie selten ein anderer: Mitglied und dabei oft Sekretär oder Präsident bei zahlreichen nationalen und internationalen Gesellschaften und in Herausgebergremien renommierter Fachjournale waren Selbstverständlichkeit; ein forderndes und mit Bravour bespieltes Extra waren die Aufgaben des medizinischen Dekanats in Innsbruck von 1993 bis 1999! Hilfe und Halt bekam der Held der Saga „ein Wiener in Tirol“ stets von seiner Ehefrau Esther und seinen Töchtern Ruth und Claire, den einzigen Personen, in deren Gegenwart sich sein "Alpha“ mühelos und automatisch in den zweiten Buchstaben des griechischen Alphabets wandelte.

Mein erster Kontakt mit PF, damals erster Oberarzt unter Prof. Klaus Wolff an der Hautklinik in Innsbruck, war 
die Frage: „Was soll ich denn lesen, um die Dermatologie zu erlernen?" Heute wäre die Antwort ganz einfach „Lesen's DEN Fritsch“. Damals bekam ich die Antwort: „Die Tante Jolesch“ oder „Der Untergang des Abendlandes in Anekdoten“" (Friedrich Torberg) und die Maghrebinischen Geschichten (Gregor von Rezzori). Als autoritätshöriger Tiroler kaufte ich die beiden Bücher noch am selben Tag, und ab dann erweiterten sich mein Verständnis und meine Liebe für die Wiener und die altösterreichische Welt enorm. Wie für viele andere war Peter für mich nicht nur ein idealer Lehrmeister in der Dermatologie, sondern auch ein unverzichtbarer Ratgeber für das Leben. Hier treffen wir nun ein zweites Mal auf das Eponym „DER Fritsch“: Es steht für den teils strengen, aber auch liebenswerten Kosmopoliten mit Wiener Wurzeln, der zum Wahltiroler (er feiert gern im Urichhaus der Kaiserjäger!) gewandelt (wurde), eine Persönlichkeit, der von seinen Freunden und Verehrern ehrfurchtsvoll mit dem Artikel der verehrungsvollen Liebkosung angesprochen wurde oder wird, eben „DER Fritsch“ (wie DER Girardi, DIE Wessely, DER Demel, DAS Sacher).

Danke für alles, Dein Helmut

H. Hintner

Salzburg

\section{Korrespondenzadresse}

\section{Prof. Dr. Helmut Hintner}

Doktor-Franz-Rehrl-Platz 2, 5020 Salzburg,

Österreich

hhintner@icloud.com

\section{Möchten Sie einen Beitrag für die Zeitschrift Der Hautarzt einreichen?}

Wir freuen uns, dass Sie unsere Zeitschrift Der Hautarzt mitgestalten möchten. Um Ihnen bei der Manuskripterstellung behilflich zu sein, haben wir für unsere Autoren Hinweise zusammen gestellt, die Sie im Internet finden unter www.DerHautarzt.de (Für Autoren).

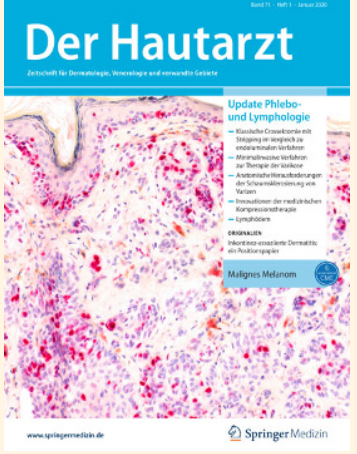

Bitte senden Sie Ihren fertigen Beitrag an:

\section{Originalien/Übersichten/}

Kasuistiken/Wie lautet Ihre Diagnose?

Prof. Dr. Daniela Bruch-Gerharz

Hautklinik des Universitätsklinikums

Düsseldorf, Moorenstr. 5,

40225 Düsseldorf

sdgehrke@googlemail.com

\section{CME Zertifizierte Fortbildung:}

Anfragen an:

PD Dr. Evelyn Gaffal

Universitätshautklinik

Universitätsklinikum Magdeburg

Labor für Experimentelle Dermatologie

Leipziger Straße 44

39120 Magdeburg

evelyn.gaffal@med.ovgu.de

Prof. Dr. Sonja Ständer

Klinik und Poliklinik für Hautkrankheiten, Universitätsklinikum Münster

Von-Esmarch-Str. 58

48149 Münster

sonja.staender@uni-muenster.de

Prof. Dr. Rolf-Markus Szeimies

Klinik für Dermatologie und Allergologie,

Knappschaftskrankenhaus Recklinghausen,
Klinikum Vest $\mathrm{GmbH}$, Dorstener Str. 151, 45657 Recklinghausen dermatologie@kk-recklinghausen.de

PD Dr. Dr. Alexander Zink

Klinik und Poliklinik für Dermatologie und Allergologie am Biederstein, Technische Universität München Biedersteiner Str. 29 80802 München alexander.zink@tum.de

Dermatoskopie - Fall des Monats: Prof. Dr. Andreas Blum Hautarztpraxis Konstanz und Lehrpraxis der Medizinischen Fakultät Universität Tübingen Augustinerplatz 7 78462 Konstanz a.blum@derma.de

\section{Rezepturtipp für die Praxis aus der} Praxis:

Bitte schreiben Sie bei Fragen an:

hautarzt@dac-nrf.de 